\title{
Contextual Information Modulates Initial Processes of Syntactic Integration: The Role of Inter- Versus Intrasentential Predictions
}

\author{
Ina Bornkessel \\ Max Planck Institute of Cognitive Neuroscience
}

\author{
Matthias Schlesewsky \\ University of Marburg
}

\author{
Angela D. Friederici \\ Max Planck Institute of Cognitive Neuroscience
}

\begin{abstract}
The authors demonstrate that intersentential context may influence syntactic integration processes during online sentence comprehension, although this influence appears to be restricted to cases in which a contextual requirement must be fulfilled. By applying event-related brain potentials to the processing of clause-medial word order variations in German, the authors show that the local processing difficulty (a negativity from 300 to $450 \mathrm{~ms}$ ) observed for object-initial sentences in a neutral context is also obtained in a (behaviorally) facilitating context in which the object is contextually given. By contrast, the processing pattern for a focused (questioned) initial object does not differ from that for a focused subject: both elicit a parietal positivity $(280-480 \mathrm{~ms})$ postonset of the focused phrase. The authors interpret this early positivity as a general marker of focus integration, a process that appears to briefly supersede sentence-internal requirements.
\end{abstract}

A recurring interest in language processing research has been the examination of possible influences of intersentential context on sentence internal parsing mechanisms. Within this line of research, most studies have focused on the question of whether contextual information may influence on-line processes of ambiguity resolution during sentence comprehension (e.g., Altmann, 1999; Altmann, Garnham, \& Dennis, 1992; Altmann, Garnham, \& Henstra, 1994; Altmann \& Steedman, 1988; Altmann, van Nice, Garnham, \& Henstra, 1998; Britt, Perfetti, Garrod, \& Rayner, 1992; Crain \& Steedman, 1985; Liversedge, Pickering, Branigan, \& van Gompel, 1998; Trueswell \& Tanenhaus, 1991; van Berkum, Brown, \& Hagoort, 1999). In this way, evidence for the application of explicit contextual information has been provided for various types of syntactic ambiguity: for example, prepositional phrase attachment ambiguities, main verb/reduced relative clause ambiguities (Altmann \& Steedman, 1988; Crain \& Steedman, 1985) and late closure ambiguities (Altmann et al., 1998) but also for the agen-

Ina Bornkessel and Angela D. Friederici, Department of Neuropsychology, Max Planck Institute of Cognitive Neuroscience, Leipzig, Germany; Matthias Schlesewsky, Junior Research Group Neurolinguistics, University of Marburg, Marburg, Germany.

The research reported here was supported by Grant FOR 375/1-4 from the Deutsche Forschungsgemeinschaft (DFG). We thank Helen de Hoop and audiences at the Universities of Salzburg and Leipzig for helpful discussions relating to this work and Ulrike Barth and Cornelia Schmidt for the data acquisition. Finally, we are grateful to two anonymous reviewers for valuable comments on previous versions of this article.

Correspondence concerning this article should be addressed to Ina Bornkessel, Max Planck Institute of Cognitive Neuroscience, Stephanstr. 1a, 04103 Leipzig, Germany. E-mail: bornke@cns.mpg.de tive/locative ambiguity of a by-phrase (Liversedge, Pickering, Branigan, \& van Gompel, 1998).

In light of experimental findings such as these, there is general agreement that contextual information plays an important role in on-line sentence comprehension, although there is no consensus regarding the time course of its application. Essentially, it is controversial whether contextual information may immediately determine syntactic parsing decisions (Altmann et al., 1992, 1994; Altmann \& Steedman, 1988; Britt et al., 1992; Crain \& Steedman, $1985)$ or whether initial syntactic parsing operations apply autonomously (i.e., independently of extrasyntactic factors such as context information; Ferreira \& Clifton, 1986; Frazier, 1987; Mitchell, Corley, \& Garnham, 1992; Murray \& Liversedge, 1994; Rayner, Garrod, \& Perfetti, 1992). In this way, the former approach assumes that misanalyses ("garden paths") may be avoided when context information is available to guide the syntactic analysis, whereas the latter posits that the apparent neutralization of garden paths in appropriate contexts is due to a post initial application of the contextual information (e.g., in terms of guidance of reanalysis processes). It is evident that dissociating between these two proposals is of fundamental importance with regard to the architecture of human sentence processing.

The present study aims to shed light on the time course of early sentence-internal and intersentential influences during language comprehension by applying an experimental method with a high degree of temporal resolution to the examination of contextual influences on word-order variations in German. As is discussed in detail below, the novelty of using these types of sentence structures is that they allow for an examination of context effects in the processing of syntactically unambiguous constructions. 


\section{Word Order and Context}

Within the theoretical syntactic literature, the order of arguments in a sentence has often been considered a locus for contextual dependencies in various languages such as Hungarian, Greek, Russian, and German (cf. Kiss, 1995, for an overview). It is important to note that, in these languages, there exist argument orders that are infelicitous when uttered in the absence of a context or in a neutral context but that may be licensed by appropriate contextual information. ${ }^{1}$ An example of a contextually licensed word order in German is given in (1), as cited from Lenerz (1977, p. 99).

(1) a. Wer ist dem Hirsch in die Flanke gesprungen? who $_{\text {NOM }}$ is [the deer $]_{\text {DAT }}$ in the flank jumped

"Who (what) jumped at the deer's flank?"

b. Ich glaube, dass dem Hirsch der Hund in die Flanke gesprungen ist. I believe that [the deer $]_{\mathrm{DAT}}$ [the $\left.\operatorname{dog}\right]_{\mathrm{NOM}}$ in the flank jumped is "I think that the dog jumped at the deer's flank."

The embedded sentence in (1b) is object-initial (OS), in other words, it departs from the default (nominative) subject-initial (SO) word order and is therefore infelicitous in a neutral context. However, this clause medial argument order variation ("scrambling") is licensed in the context of a sentence such as (1a). Specifically, the question-answer pair in (1) shows that an object that is a topic (i.e., given by the context) can felicitously appear before the subject clause medially. ${ }^{2}$

In the absence of a context, OS orders as in (1b) have been shown to elicit a measurable increase in (on-line) processing load at the position of the fronted object (Bornkessel, Schlesewsky, \& Friederici, 2002; Rösler, Pechmann, Streb, Röder, \& Hennighausen, 1998; Schlesewsky, Bornkessel, \& Frisch, 2003). This effect has been interpreted as reflecting a local mismatch between the predicted subject position and the object encountered in this position (Friederici, Schlesewsky, \& Fiebach, 2003). Because, as described above, information structure (i.e., a topic context) supposedly licenses OS orders, approaches assuming an initial influence of context on basic syntactic operations would predict a modulation of the processing difficulty observed for OS structures when these are embedded in a suitable context.

Previous behavioral findings appear to confirm this prediction. Thus, Meng, Bader, and Bayer (1999) reported a study in which direct question contexts leading to a topic interpretation of an initial object (clause-medially) neutralized the enhanced processing cost observed for OS sentences in a neutral context in terms of both reading times and acceptability ratings. This study therefore showed that there is a contextual influence on the processing of OS sentences in German. However, Meng et al. only reported reading times for the sentence-final position (an auxiliary) in their sentences, thereby precluding all conclusions with regard to the precise point in time at which the contextual support applies. Because time-sensitive measures of clause-medial word-order variations indicate that the processing difficulty in these structures results from local processes at the position of the fronted constituent, Meng et al.'s study leaves open the crucial question of whether context may modulate these local processing difficulties.

\section{Electrophysiological Measures of Processing Difficulties in OS Sentences}

Evidence for the local nature of the processing difficulty in clause-medial word-order variation in German (e.g., 2) stems primarily from studies using the event-related brain potentials (ERPs) methodology. ${ }^{3}$

(2) Vielleicht hatte den Brief der Lehrer dem Gärtner gezeigt. perhaps had [the letter $]_{\mathrm{ACC}}[\text { the teacher }]_{\mathrm{NOM}}[\text { the gardener }]_{\mathrm{DAT}}$ shown "Perhaps the teacher had shown the letter to the gardener."

For sentences such as (2), Rösler et al. (1998) and Schlesewsky et al. (2003) observed a negative deflection with a left focus in the ERP elicited by the initial accusative object, den Brief ("the letter"), compared with a nominative subject in the same position. This effect was observable between 300 and $450 \mathrm{~ms}$ postonset of the determiner den ("the ${ }_{\mathrm{ACC}}$ "), the initial positioning of which unambiguously signals a noncanonical word order in a neutral context. A similar ERP pattern is obtained in embedded sentences introduced by the complementizer dass ("that"; Bornkessel et al., 2002), thus indicating that the left negativity between 300 and $450 \mathrm{~ms}$ is a general effect elicited by clause-medial word-order variations. On the basis of the behavioral experiments showing a sentence-final influence of context on word order, psycholinguistic models assuming an initial influence of context on syntactic parsing operations would predict a modulation of the local negativity elicited by word-order variations in a facilitating context. Proponents of autonomous syntactic approaches, by contrast, would assume no such influence.

\section{The Present Study}

The present study examines whether context modulates the local processing difficulties observed for clause-medial word-order variations in German. In keeping with previous studies (e.g., Altmann et al., 1998; Meng et al., 1999), we used target sentences following a context question. In addition to allowing for maximally con-

\footnotetext{
${ }^{1}$ Although this type of context influence has been discussed for many languages, we focus on German in the following, because most of the other languages in question have not been subjected to basic psycholinguistic investigations of argument order variations (with the notable exception of Keller \& Alexopoulou's, 2001, study for Greek).

${ }^{2}$ Although there are conflicting views on the proper linguistic definition of topic, we adopt the position that a topic is simply a contextually given constituent for the sake of simplicity.

${ }^{3}$ ERPs are small changes in the spontaneous electrical activity of the brain that occur in response to certain sensory or cognitive stimuli and that may be recorded continuously and noninvasively by means of electrodes attached to the scalp. The recording of ERPs has proven particularly fruitful for the examination of complex linguistic phenomena, because this method not only provides a very high degree of temporal resolution but also allows qualitatively different processes to be differentiated in terms of various dimensions of the ERP signal (e.g., latency, topography, and polarity, i.e., negativity vs. positivity; e.g., Friederici, 1999, 2002; Hagoort, Brown, \& Groothusen, 1993; Kutas \& Hillyard, 1980, 1983; Neville, Nicol, Barss, Forster, \& Garrett, 1991; Osterhout \& Holcomb, 1992, 1993 , among many others).
} 
trolled contextual influences, this type of context also enabled us to examine the influence of contextual factors in three different dimensions, namely with regard to the role of givenness and contextual predictions as well as to the precise nature of these predictions. In the following sections, we discuss these three domains of examination and formulate hypotheses for each. All experimental conditions referred to in the following are exemplified in Table 1 -note that SO and OS target sentences are identical across conditions.

Table 1

Example Context and Target Sentences for Each of the Critical Conditions in the Present Study

\begin{tabular}{ll}
\hline Condition & Example \\
\hline
\end{tabular}

NEUT (context)

Klaus fragt sich, was am Sonntag passiert ist. Klaus asks himself what on Sunday happened is.

SO-TOP (context) Klaus fragt sich, wen der Gärtner am Sonntag besucht hat.

Klaus asks himself who ${ }_{A C C}$ [the gardener] $]_{N O M}$ on Sunday visited has.

SO-FOC (context) Klaus fragt sich, wer am Sonntag den Lehrer besucht hat.

Klaus asks himself who ${ }_{N O M}$ on Sunday [the teacher $]_{A C C}$ visited has.

SO-MMC (context) Klaus fragt sich, wen der Lehrer am Sonntag besucht hat.

Klaus asks himself who ACC $\left._{\text {[the teacher }}\right]_{N O M}$ on Sunday visited has.

SO-MML (context) Klaus fragt sich, wer am Sonntag den Gärtner besucht hat.

Klaus asks himself who ${ }_{\text {NOM }}$ on Sunday [the gardener] ${ }_{A C C}$ visited has.

SO target Dann erfuhr er, dass der Gärtner den Lehrer besucht hat.

Then heard he that [the gardener] $]_{\text {NOM }}$ [the teacher $]_{A C C}$ visited has.

OS-TOP (context) Klaus fragt sich, wer am Sonntag den Gärtner besucht hat.

Klaus asks himself who ${ }_{\text {NOM }}$ on Sunday [the gardener $]_{A C C}$ visited has.

OS-FOC (context) Klaus fragt sich, wen der Lehrer am Sonntag besucht hat.

Klaus asks himself who ${ }_{A C C}$ [the teacher] $]_{N O M}$ on Sunday visited has.

OS-MMC (context) Klaus fragt sich, wer am Sonntag den Lehrer besucht hat.

Klaus asks himself who ${ }_{\text {NOM }}$ on Sunday [the teacher] $]_{A C C}$ visited has.

OS-MML (context) Klaus fragt sich, wen der Gärtner am Sonntag besucht hat.

Klaus asks himself who ${ }_{A C C}$ [the gardener] $]_{N O M}$ on Sunday visited has.

OS target

Dann erfuhr er, dass den Gärtner der Lehrer besucht hat.

Then heard he that [the gardener] $]_{A C C}[$ the teacher $]_{N O M}$ visited has.

Note. $\quad \mathrm{SO}=$ subject-initial $; \mathrm{OS}=$ object-initial; $\mathrm{NEUT}=$ neutral context; TOP $=$ NP1 (Noun Phrase 1 ) is given by the context; FOC $=$ NP1 is focused; $\mathrm{MMC}=\mathrm{NP} 1$ case mismatch between the $w h$-pronoun and NP1; MML $=$ lexical mismatch between the $w h$-pronoun and NP1. All translations are word by word.

\section{Givenness (Object $=$ Topic)}

As shown by Meng et al. (1999), OS orders in German are more acceptable when the fronted object is a topic. In the present experiment, our goal was to determine whether a topic (TOP) context modulates the negativity typically observed on initial (clause-medial) objects (i.e., whether a globally facilitating context may also aid initial syntactic integration processes). If this is the case, an initial object that is a topic (condition OS-TOP in Table 1) should elicit a reduced negativity compared with an initial object in a neutral context (OS-NEUT). The corresponding control conditions are the condition in which the initial subject is a topic (SO-TOP) and the neutral SO condition (SO-NEUT).

\section{Contextual Predictions (Focus)}

On the basis of a number of experimental studies, Altmann et al. (1998) argued that the influence of question contexts differs from that of nonquestion contexts, because only the former generate contextual predictions. Thus, an answer to a question such as, "Who visited Max?", must minimally include an element filling the slot opened by the wh-pronoun (e.g., "Peter"). A sentence not fulfilling this expectation is an infelicitous answer to the question. Altmann et al.'s (1998) data suggested that exactly these types of contextual requirements - and, more specifically, the expectations generated by them-allow for default parsing strategies to be overridden in ambiguous structures. This suggests that questions provide a way of inducing contextual override that other contexts may lack.

The present study allowed us to investigate the time course of contextually induced predictions in unambiguous sentences. In this regard, consider Condition SO-focus (-FOC) in Table 1. Here, the wh-pronoun wer ("who ${ }_{\mathrm{NOM}}$ ") generates an expectation that the target sentence will introduce a new referent realized by a nounphrase [NP] bearing nominative case. In linguistic terms, the question focuses (FOC) the constituent for which it is asking. This prediction is borne out when der Gärtner ("[the gardener $]_{\mathrm{NOM}}$ ") is encountered in the target sentence. Most interesting in this regard is, of course, the question of how such intersentential predictions interact with sentence-internal processing requirements (e.g., the prediction of a subject position discussed above). At the position of den Gärtner ("[the gardener] $]_{\mathrm{ACC}}$ ") in the target sentence of the OS-FOC condition, there is a conflict between intersentential and sentence-internal processing requirements: Although this argument fulfills the requirement of the contextual prediction for an accusative-marked NP, it does not match the intrasentential requirement for a subject. Constructions such as OS-FOC therefore allow us to examine how intersentential and intrasentential processing requirements interact.

Because contextual predictions have not been previously examined using ERPs, it is impossible to formulate clear predictions with regard to the component(s) to be expected when a predicted element is processed. However, if there is a general effect of contextual integration, this should be observable independent of the position in the sentence where the expected element appears (i.e., in the SO/OS-TOP conditions, where NP1 is given, contextual integration should take place at the position of NP2). Hence, to examine possible effects beyond the position of NP1, we also analyze ERPs for NP2 and the verb. 


\section{The Nature of the Prediction (Lexical Vs. Case Requirements)}

Finally, the present experiment also examines the exact nature of the expectation generated by the context and its interaction with the target sentence. Essentially, in the case of a constituent question asking for an NP, the expectation generated is twofold, as we illustrate by means of the conditions SO-FOC, SO-MML, and SO-MMC in Table 1.

Whereas the target sentence in the SO-FOC condition is a perfect answer to the context question, the target sentences in both SO-MML and SO-MMC violate parts of the expectation generated by their respective context question. In SO-MML, the referent of the NP-bearing nominative case (der Gärtner) is the same as the referent referred to in the context question (den Gärtner); we refer to this condition as inducing a lexical mismatch (MML) with regard to the contextually generated expectation for a referent that is distinct from the one in the context question. In SO-MMC, by contrast, the referent of NP1 in the target sentence (der Gärtner) is new (here, the referent introduced in the context question is der Lehrer), though bearing a different case to the one predicted by the wh-pronoun of the context question (accusative rather than nominative); this condition is referred to as inducing a case mismatch (MMC) with regard to the contextual prediction. In both cases, the mismatch may be realized through an SO sentence (SO-MML/SOMMC) or through an OS sentence (OS-MML/OS-MMC). ${ }^{4}$ In this way, contrasting the ERP responses at the position of NP1 for the FOC, MML and MMC conditions allows us to shed light on whether the two parts of the contextual prediction (i.e., case and lexical content) are equally important in fulfilling the prediction, as a condition containing the critical feature for contextual integration should pattern with the normal focus condition.

\section{Method}

Participants. Twenty-four students of the University of Leipzig were paid to participate in the experiment ( 14 women; $M$ age $=24.7$ years; age range $=19-31$ years)

Materials. The critical sentences for this experiment were created on the basis of 120 noun-noun-verb triplets, which were each combined with a proper noun (60 male /60 female) and a temporal or locative prepositional phrase to form 120 sets of the 10 conditions shown in Table 1 . All nouns were two to three syllables in length and of masculine syntactic gender to allow for unambiguous case marking. To exclude lexical effects of presenting certain nouns in certain positions, a second set of sentences was generated by reversing the order of the two NPs in the target sentence and interchanging the NPs included in the context sentence. The 2,400 sentence pairs thus obtained were assigned to 6 lists of 400 sentence pairs (i.e., 40 per condition) such that each participant saw 2 sentences from the same set, 1 of which was presented in the first and the other of which was presented in the second experimental session (see below). The critical sentences were randomly interspersed with an equal number of filler contexts and targets.

Procedure. Sentences were presented visually in the center of a computer screen, with context sentences presented as a whole and target sentences presented in a phrase-by-phrase manner (i.e., determiners and nouns were presented together). Participants read the context sentences at their own pace and pressed a button to induce the presentation of the target sentence. For the target sentences, single words were presented for $450 \mathrm{~ms}$ and phrases for $500 \mathrm{~ms}$ with an interstimulus interval of $100 \mathrm{~ms}$. Following the presentation of a target sentence, participants were required to (a) judge the acceptability of this sentence within a 2,000-ms time limit and (b) complete a probe detection task in which single words from either the context or the target sentence were presented (also within a 2,000-ms time limit).

Participants were asked to avoid movements and to not blink their eyes during the presentation of the target sentence. Each participant completed two experimental sessions separated by at least a week, each of which began with a short training session followed by eight experimental blocks comprising 45 sentence pairs each. Between the blocks, participants took short breaks. Each experimental session (including electrode preparation) lasted approximately $3 \mathrm{hr}$.

The EEG was recorded by means of $43 \mathrm{AgAgCl}$ electrodes fixed at the scalp by means of an elastic cap (Electro Cap International, Eaton, $\mathrm{OH}$ ). The ground electrode was positioned above the sternum. Recordings were referenced to the left mastoid, but rereferenced to linked mastoids offline. The electrooculogram (EOG) was monitored by means of electrodes placed at the outer canthus of each eye for the horizontal EOG and above and below the participant's right eye for the vertical EOG. Electrode impedances were kept below $5 \mathrm{kOhm}$.

All EEG and EOG channels were amplified using a Twente Medical Systems (Enschede, The Netherlands) DC amplifier and recorded continuously with a digitization rate of $250 \mathrm{~Hz}$. The plots of grand average ERPs were smoothed off-line with a $10-\mathrm{Hz}$ low-pass filter, but all statistical analyses were computed on unfiltered data.

Average ERPs were calculated per condition per participant from the onset of each critical stimulus item (i.e., NP1, NP2, and the verb) to 1,000 ms postonset, before grand averages were computed over all participants. Averaging took place relative to a baseline interval from -200 to $0 \mathrm{~ms}$ before the onset of the first NP. ${ }^{5}$ Trials for which the probe detection task was not performed correctly were excluded from the averaging procedure, as were trials containing ocular, amplifier-saturation, or other artifacts (the EOG rejection criterion was $40 \mu \mathrm{V}$ ). On average, $10.75 \%$ of the experimental trials were rejected in this manner $(6.86 \%$ on the basis of the probe detection task and $3.89 \%$ due to artifacts). These were distributed equally across all conditions.

Data analysis. For the statistical analysis of the ERP data, repeated measures analyses of variance (ANOVAs) were calculated for mean amplitude values per time window per condition. The statistical analysis was carried out in a hierarchical manner, in other words, only significant interactions $(p=.05)$ were resolved. In addition, no main effects of or interactions exclusively between topographical factors are reported. To avoid excessive Type I errors due to violations of sphericity, we applied the correction of Huynh and Feldt (1970) when the analysis involved factors with more than one degree of freedom in the numerator. In these cases, we report the original degrees of freedom and the corrected probability level. For post hoc planned comparisons, the probability level was adjusted according to the modified Bonferroni procedure (cf. Keppel, 1991)

\footnotetext{
${ }^{4}$ Note, however, that, although we have described the MML and MMC conditions as inducing a mismatch, these conditions are not true violations in the sense that they are infelicitous in the context of their respective questions. For both conditions there is the possibility of a contrastivefocused reading that is approximately equivalent to the cleft construction in English (e.g., "Steve wondered who had been watching the gardener -Then he heard that it was the GARdener who had been watching the HUNter.").

${ }^{5}$ In view of the predictions of critical effects at the positions of both arguments, the pre-NP1 baseline was also used for the averages of NP2 and the verb.
} 
Topographical factors were chosen as follows. For the midline electrodes, the factor Electrode included the eight electrodes AFZ, FZ, FCZ, $\mathrm{CZ}, \mathrm{CPZ}, \mathrm{PZ}, \mathrm{POZ}$, and $\mathrm{OZ}$ as levels; for the lateral electrodes, the factors Hemisphere (HEMI; left vs. right) and Region (REGION; anterior, central, posterior) were fully crossed, thus giving rise to the following six lateral regions of interest (ROIs): left-anterior (F7, F5, F3, FT7, FC5, FC3), left-central (T7, C5, C3, TP7, CP5, CP3), left-posterior (P7, P5, P3, PO7, $\mathrm{PO} 3, \mathrm{O} 1)$, right-anterior ( $\mathrm{F} 8, \mathrm{~F} 6, \mathrm{~F} 4, \mathrm{FT} 8, \mathrm{FC} 6, \mathrm{FC} 4)$, right-central (T8, $\mathrm{C} 6, \mathrm{C} 4, \mathrm{TP} 8, \mathrm{CP} 6, \mathrm{CP} 4)$, and right-posterior (P8, P6, P4, PO8, PO4, O2).

\section{Results}

Figures 1-4 show grand-average ERPs at selected electrode positions spanning the entire clause from the onset of NP1 to 1,000 ms postonset of the clause-final verb. Figures 1 and 2 contrast sentences in a neutral context (NEUT) with sentences in which
NP1 was a topic (i.e., given by the context-TOP) and sentences in which NP1 was focused (i.e., asked for by the context-FOC) for SO and OS sentences, respectively.

In both SO (Figure 1) and OS sentences (Figure 2), focused NPs elicited a broadly distributed positivity between approximately 300 and $500 \mathrm{~ms}$ postonset of the critical constituent compared with the conditions in which the constituent was not focused: This effect was apparent relative to the position of the first argument in the $\mathrm{SO} / \mathrm{OS}-\mathrm{FOC}$ conditions and relative to the position of the second argument in the SO/OS-TOP conditions. Recall that in the latter condition, the context question focuses the second NP of the target sentence as a by-product of rendering the initial NP a topic. For the OS sentences, Condition OS-TOP gives rise to a fronto-central negativity between approximately 350 and $450 \mathrm{~ms}$ postonset of NP1 compared with OS-NEUT. Therefore, it appears that given-
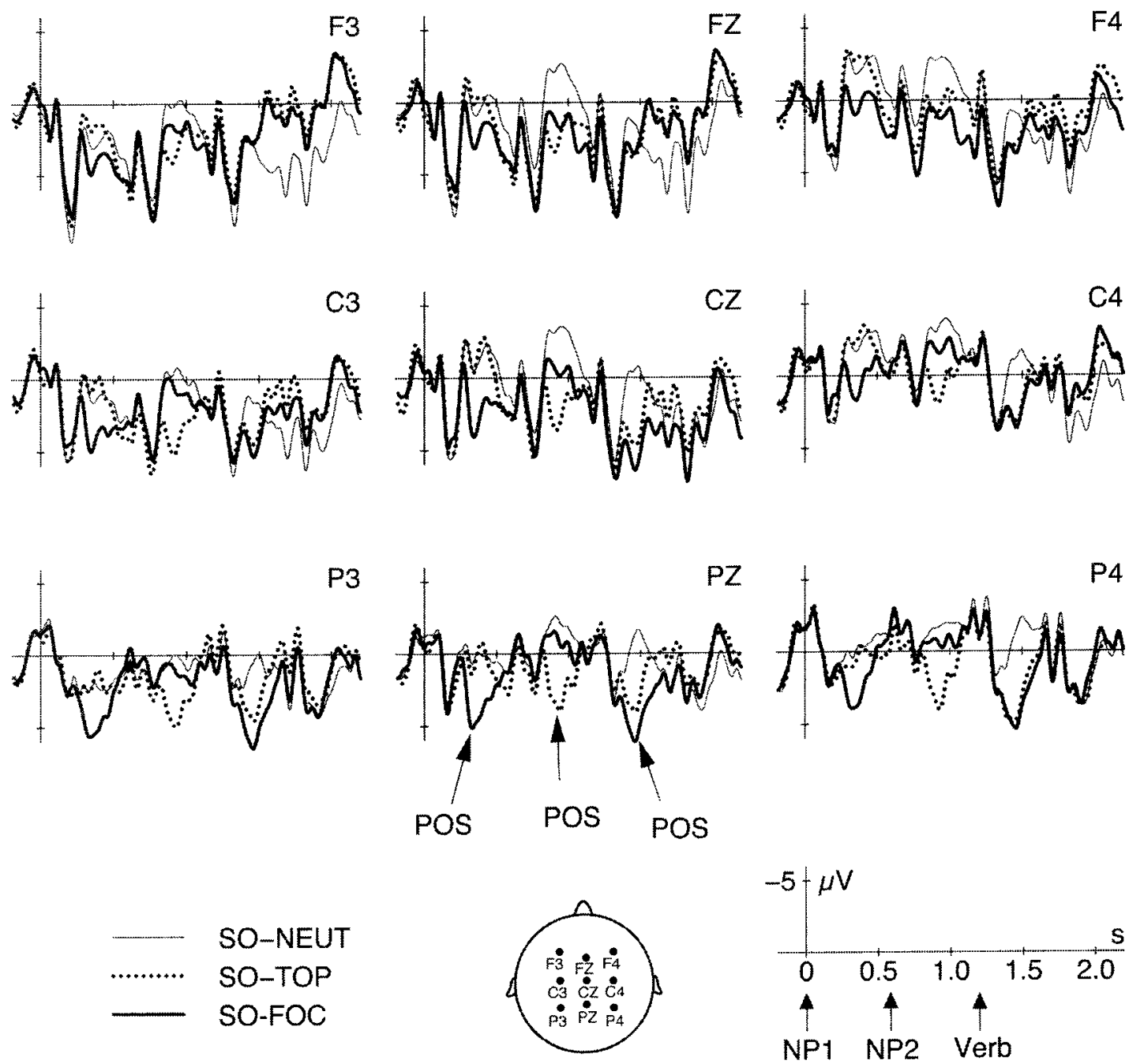

Figure 1. Grand average event-related potentials (ERPs) at selected electrode positions for subject-initial (SO) sentences from the onset of Noun Phrase 1 (NP1) to 1,000 ms postonset of the verb in a neutral context (SO-NEUT), a focusing context (SO-FOC), and a topic context (SO-TOP). Negativity is plotted upward. POS $=$ positivity. 

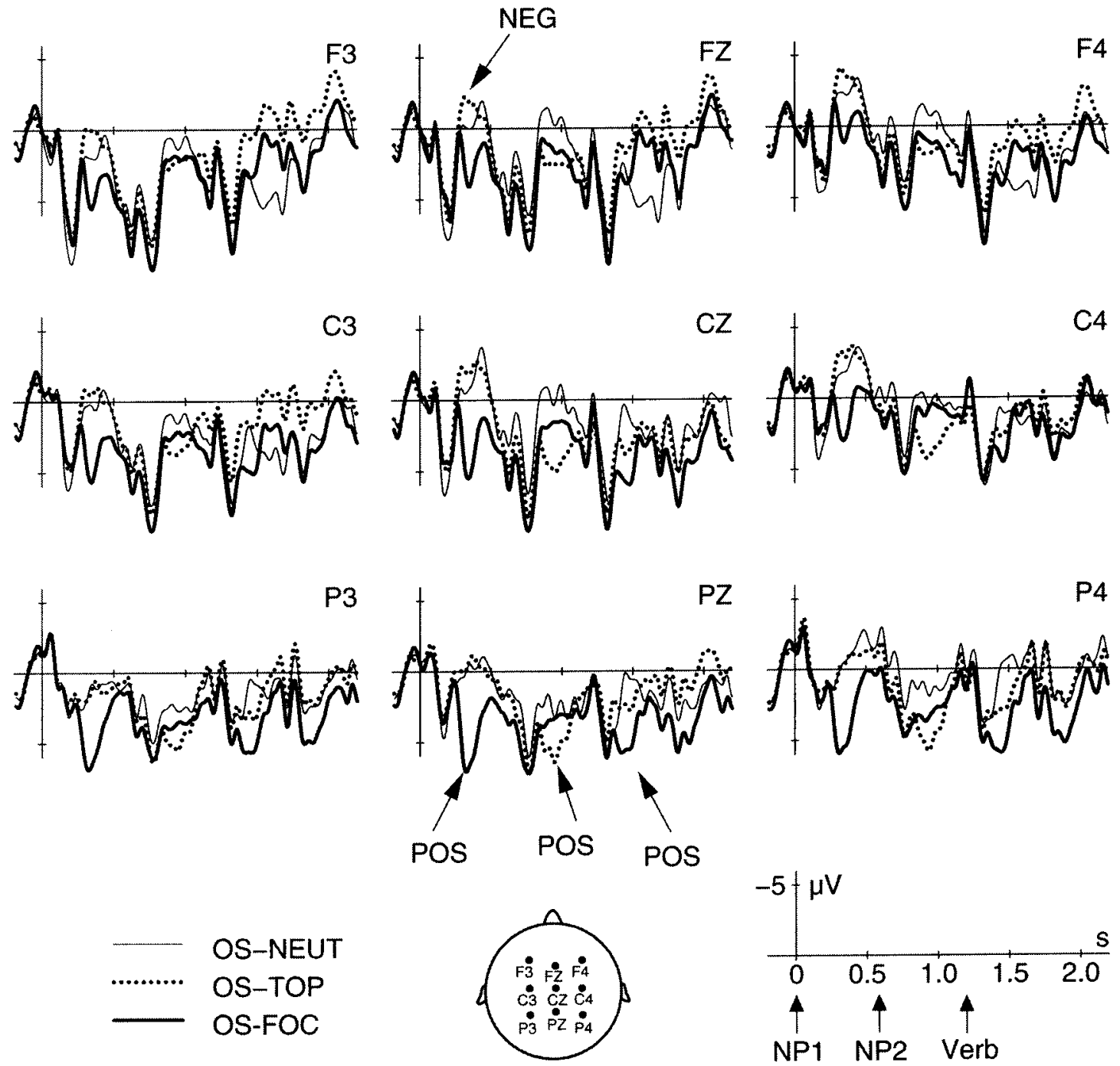

Figure 2. Grand average event-related potentials (ERPs) at selected electrode positions for object-initial (OS) sentences from the onset of Noun Phrase 1 (NP1) to 1,000 ms postonset of the verb in a neutral context (OS-NEUT), a focusing context (OS-FOC), and a topic context (OS-TOP). Negativity (NEG) is plotted upward. POS $=$ positivity.

ness of the initial object enhances rather than reduces the scrambling negativity.

The comparisons between the NEUT and MM conditions are shown in Figures 3 and 4 for SO and OS sentences, respectively.

As Figures 3 and 4 show, only the MMC conditions (i.e., the conditions in which NP1 introduces a new referent, although case-marked differently to the contextually predicted constituent) give rise to positivities at the position of NP1. Visual inspection of the MMC conditions indicates that the positivity for this condition is larger in the SO than in the OS case. For the MML conditions, in which NP1 bears the case-marking predicted by the context but does not introduce a referent distinct from the one in the context question, no effect is apparent at the position of NP1 in the SO case. In the case of OS sentences, however, the neutral condition shows a negativity at left central and posterior sites compared with the MML condition at the position of NP1. At the position of NP2, the MML conditions behave similarly to the TOP conditions in that they elicit a positivity. Finally, at the position of the verb, all nonneutral conditions show an early positivity relative to the neutral conditions irrespective of word order.

In view of the effects revealed by visual inspection of the grand averages, we chose two time windows for the statistical analysis: (a) 280-480 ms postonset of NP1, NP2, and the verb for the positivity for focused constituents (NPs) and nonneutral contexts (verb), and (b) 350-550 ms postonset of NP1 for the negativity for OS sentences. Note that in the following, all planned comparisons reported as significant are significant at at least the probability 

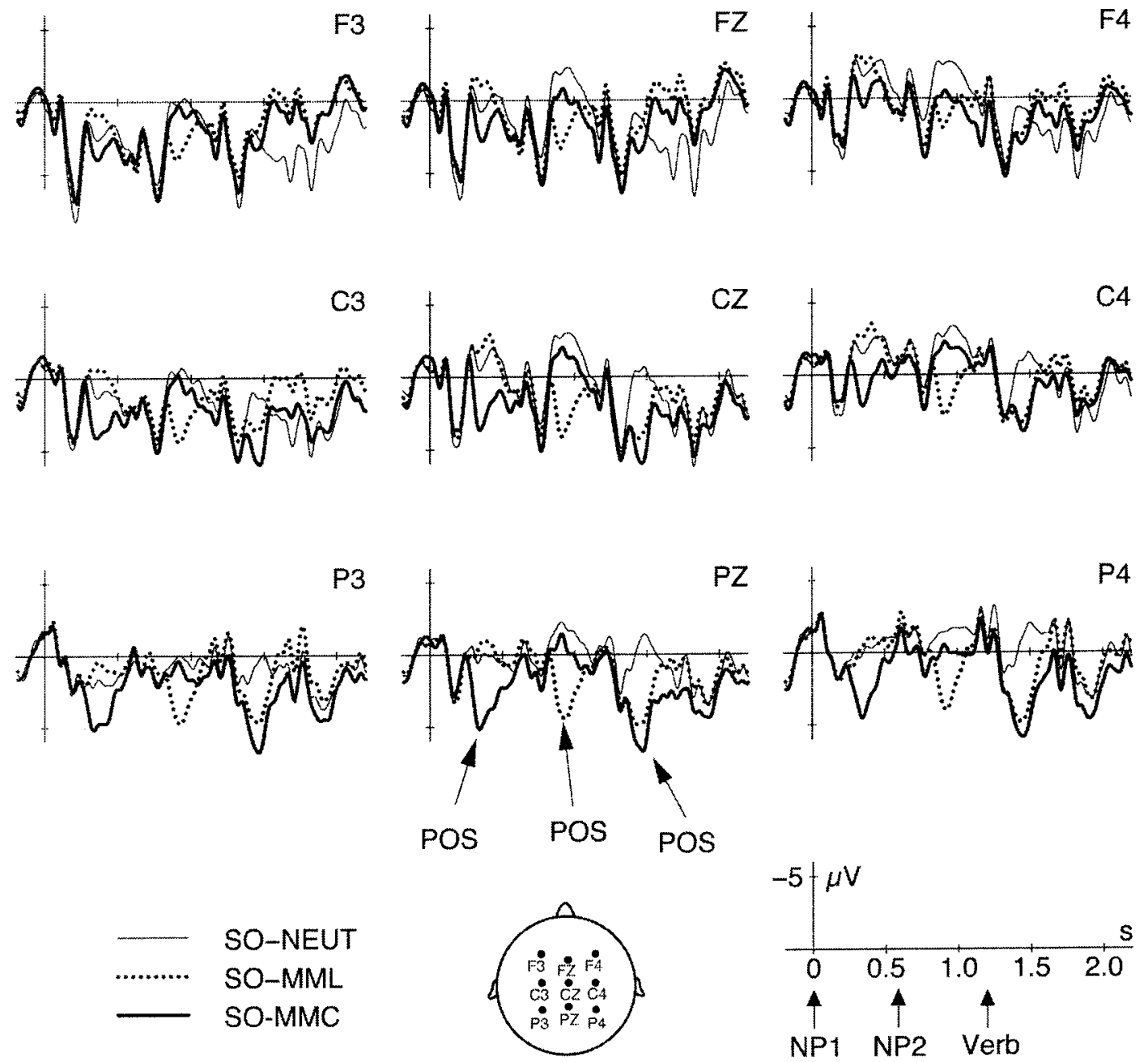

Figure 3. Grand average event-related potentials (ERPs) at selected electrode positions for subject-initial (SO) sentences from the onset of Noun Phrase 1 (NP1) to 1,000 ms postonset of the verb in a neutral context (SO-NEUT), a lexical mismatch context (SO-MML), and a case mismatch context (SO-MMC). Negativity is plotted upward. POS $=$ positivity.

level of .05. The complete statistical analysis of the ERP and behavioral data for this study as well as mean amplitude differences for all significant effects are presented at http://www .cns.mpg.de/Projects/syn_context.

\section{Early Positivity: NP1 (280-480 Ms)}

The reliability of the positivity for the FOC and MMC conditions at the position of NP1 was confirmed by the statistical analysis in the time window $280-480 \mathrm{~ms}$ postonset of this constituent. The analysis of the lateral electrodes revealed a significant main effect of CONTEXT, $F(4,92)=27.80, p<.01$, $M S E=4.87$, and an interaction CONTEXT $\times$ REGION, $F(8$, $184)=6.77, p<.01, M S E=0.71$. Separate analyses for each of the six ROIs defined above revealed significant effects of
CONTEXT in all ROIs. Planned comparisons between individual contexts showed that ERP responses to FOC and MMC were more positive than NEUT in central and posterior regions.

With regard to the midline electrodes, the global statistical analysis also showed a main effect of CONTEXT, $F(4$, $92)=28.20, p<.01, M S E=12.90$, as well as interactions ORDER $\times$ CONTEXT, $F(4,92)=3.35, p<.05, M S E=11.59$, and CONTEXT $\times$ ELECTRODE, $F(28,644)=7.07, p<.01$, $M S E=0.84$. Both SO and OS structures showed main effects of CONTEXT and single comparisons for individual contexts within both word orders revealed that FOC was more positive than NEUT in both word orders as was MMC compared with NEUT in SO sentences. For OS orders, FOC was significantly more positive than MMC, thus confirming the descriptive impression that the early positivity is reduced in the OS-MMC condition relative to the OS-FOC condition. 

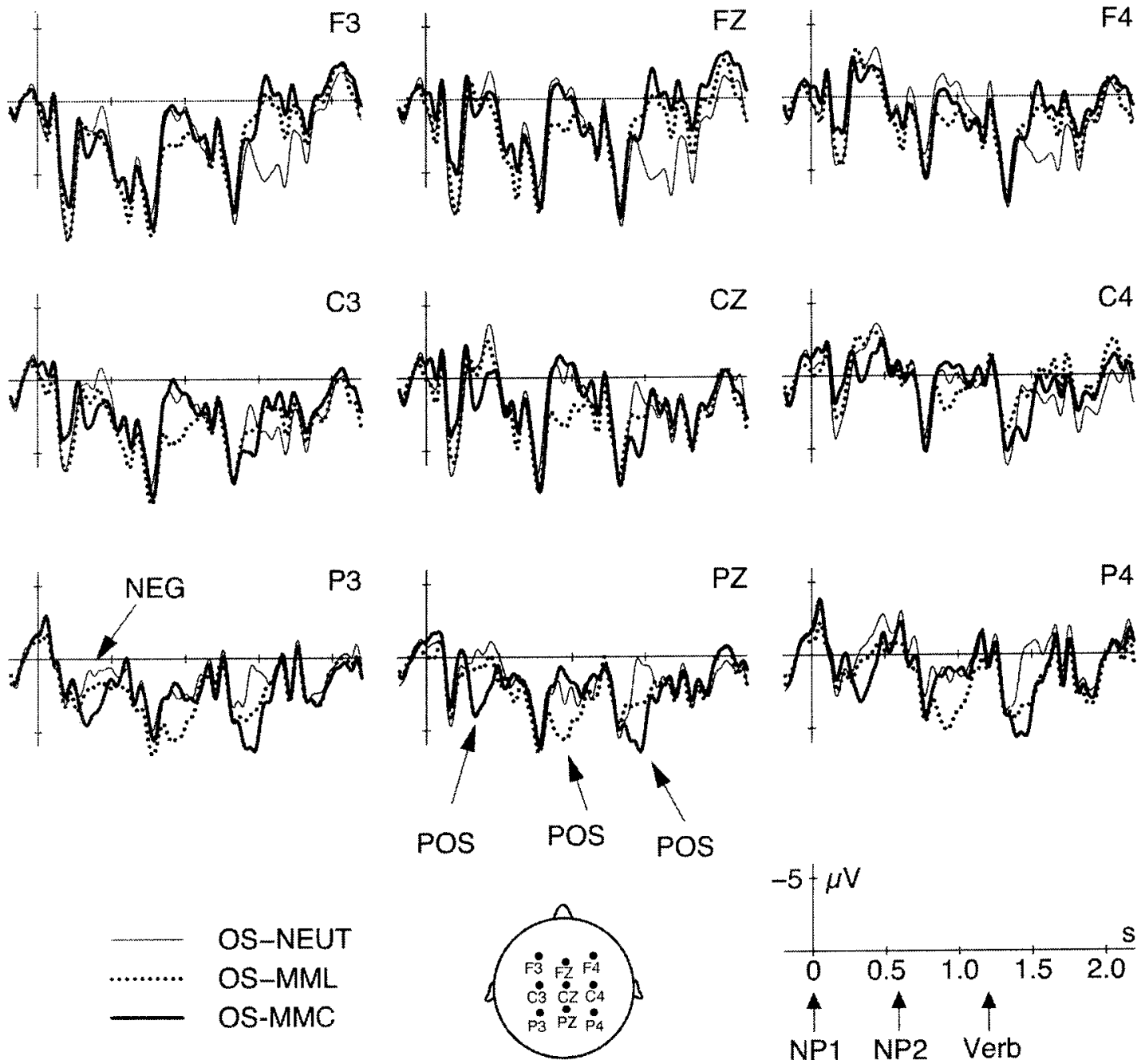

Figure 4. Grand average event-related potentials (ERPs) at selected electrode positions for object-initial (OS) sentences from the onset of Noun Phrase 1 (NP1) to 1,000 ms postonset of the verb in a neutral context (OS-NEUT), a lexical mismatch context (OS-MML), and a case mismatch context (OS-MMC). Negativity is plotted upward. POS $=$ positivity.

\section{Early Positivity: NP2 (280-480 Ms)}

At the position of NP2, the TOP and MML conditions showed positivities compared with NEUT. The global statistical analysis for the lateral electrodes showed a main effect of CONTEXT, $F(4$, $92)=13.50, p<.01, M S E=8.63$, as well as interactions of CONTEXT $\times$ HEMI, $F(4,92)=2.94, p<.05, M S E=1.24$, and ORDER $\times$ CONTEXT $\times$ HEMI, $F(4,92)=2.57, p<.05$, $M S E=0.78$. Analyses per ROI revealed significant effects of CONTEXT in all regions, with TOP and MML always significantly more positive than NEUT.

The global analysis of the midline electrodes also revealed main effects of CONTEXT, $F(4,92)=14.54, p<.01, M S E=23.98$, and an interaction CONTEXT $\times$ ELECTRODE, $F(28$, $644)=2.14, p<.05, M S E=1.96$. Single comparisons for the individual electrodes showed effects of CONTEXT at all electrode sites, with TOP and MML more positive than NEUT.

\section{Early Positivity: Verb (280-480 Ms)}

At the position of the verb, visual inspection revealed positivities for all nonneutral conditions compared with the neutral conditions. The global statistical analysis for the lateral electrodes showed a main effect of CONTEXT, $F(4,92)=2.66$, $p<.05, M S E=21.18$, and interactions of CONTEXT $\times$ HEMI, $F(4,92)=8.11, p<.01, M S E=1.40$, and CONTEXT $\times$ REGION, $F(8,184)=20.17, p<.01, M S E=1.88$. The effects of CONTEXT reached significance in posterior and left-anterior ROIs. Planned comparisons between individual contexts in the ROIs showing a significant effect of CONTEXT revealed that the four nonneutral conditions (TOP, FOC, MML, and MMC) were all more positive than the NEUT conditions in posterior regions.

With regard to the midline electrodes, the global statistical analysis revealed a main effect of CONTEXT, $F(4,92)=3.54$, 
$p<.05, M S E=50.28$, and an interaction of CONTEXT $\times$ ELECTRODE, $F(28,644)=9.61, p<.01, M S E=2.46$. Analyses for individual electrodes showed significant effects of context from $\mathrm{CZ}$ to OZ. Planned comparisons for individual contexts at the electrodes showing a main effect of CONTEXT revealed that FOC and MMC were reliably more positive than NEUT from CPZ-OZ, MML from POZ-OZ, and TOP at OZ.

\section{Scrambling Negativity: NP1 (350-550 Ms)}

In the time window $350-550 \mathrm{~ms}$ relative to the onset of NP1, OS structures showed a negativity compared with their SO counterparts in NEUT, TOP, and MMC contexts (in the latter, in the form of a reduced positivity). For the sake of conciseness, we report only effects related to word order for this time window, because pure context effects likely stem from a partial overlap with the time window for the early positivity at the position of NP1.

The global statistical analysis in the time window 350-550 ms revealed an interaction of ORDER $\times$ CONTEXT, $F(4,92)=2.86$, $p<.05, M S E=4.11$, as well as a marginally significant interaction of ORDER $\times$ CONTEXT $\times$ REGION, $F(8,184)=2.06, p<$ $.07, M S E=0.32$. Separate analyses in the six ROIs revealed significant interactions ORDER $\times$ CONTEXT in left-central, left-posterior, and right-posterior regions. OS conditions were significantly more negative than SO conditions in the following regions and contexts: left-central (NEUT, TOP, and MMC), leftposterior (NEUT and MMC), and right-posterior (TOP and MMC).

The global analysis of the midline electrodes revealed a significant interaction ORDER $\times$ CONTEXT, $F(4,92)=3.60, p<.05$, $M S E=10.57$. Separate analyses for the individual contexts revealed more negative waveforms for OS in comparison to SO conditions for the TOP and MMC contexts.

Finally, to test the hypothesis that the negativity for the OS-TOP condition (i.e., the condition in which the first NP is given by the context) indeed has a shorter latency than the negativity in the OS-NEUT condition, we conducted planned comparisons between these two conditions over the midline electrodes in two 100-ms windows (350-450 $\mathrm{ms}$ and $450-550 \mathrm{~ms})$. The analysis in the 350-450-ms time window revealed a significant interaction of CONTEXT $\times$ ELECTRODE, $F(7,161)=3.70, p<.05$, $M S E=1.00$. Resolving this interaction by ELECTRODE showed a significant negativity for OS-TOP compared with OS-NEUT at the electrodes AFZ, FZ, and FCZ. In the time window 450-550 $\mathrm{ms}$, by contrast, no significant differences emerged.

\section{Discussion}

With regard to the question of whether context may influence initial processes of syntactic integration, the present experiment showed two basic effects: (a) a positivity (280-480 ms) for elements predicted by the constituent (context) question and on the verb in nonneutral contexts and (b) a negativity (350-550 ms) on the first NP in OS sentences in a neutral context or a topic context (though with a shorter latency for the latter). A schematic summary of the experimental conditions and the effects elicited by them at the position of NP1 is given in Table 2 .

The columns under Prediction in Table 2 show the intra- and intersentential predictions applying to the position of NP1. With regard to the former, only the prediction of a nominative-marked argument (subject) is relevant. The intersentential predictions, by contrast, refer to those properties required to fulfill the contextual requirement induced by the $w h$-pronoun in the context question with respect to (a) a referent distinct from that given in the context question (as realized by a new lexical item, "-lex") and (b) the case marking required to fill the slot opened by the wh-phrase $(+$ nom/ + acc). The Constituent Encountered columns describe the actual properties of NP1 in each of the conditions. Thus, Table 2 provides an overview of which conditions fulfill and which conditions violate intra- and intersentential requirements (predictions) at the position of NP1 in addition to showing which ERP patterns were observable. In the following, we discuss first the pattern of results for SO structures, before turning to the OS sentences.

Table 2

Schematic Summary of the Conditions in the Present Experiment and the Components Elicited by Them at the Position of NP1

\begin{tabular}{|c|c|c|c|c|c|c|}
\hline \multirow[b]{3}{*}{ Condition } & \multicolumn{3}{|c|}{ Prediction } & & & \multirow[b]{3}{*}{ Component } \\
\hline & \multirow[b]{2}{*}{ Intrasent. } & \multicolumn{2}{|c|}{ Intersent. } & \multicolumn{2}{|c|}{ Constituent encountered } & \\
\hline & & Case & Lex & Case & Lex & \\
\hline SO-NEUT & + nom & - & - & + nom & - & - \\
\hline SO-TOP & + nom & + acc & $-\operatorname{lex}$ & + nom & + lex & - \\
\hline SO-FOC & + nom & + nom & $-\operatorname{lex}$ & + nom & $-1 \mathrm{ex}$ & Early positivity \\
\hline SO-MMC & + nom & $+\mathrm{acc}$ & $-\operatorname{lex}$ & + nom & $-\operatorname{lex}$ & Early positivity \\
\hline SO-MML & + nom & + nom & $-\operatorname{lex}$ & + nom & + lex & - \\
\hline OS-NEUT & + nom & - & - & + acc & - & Negativity \\
\hline OS-TOP & + nom & + nom & $-1 \mathrm{ex}$ & + acc & + lex & Negativity \\
\hline OS-FOC & + nom & $+\mathrm{acc}$ & $-\operatorname{lex}$ & $+\mathrm{acc}$ & $-1 \mathrm{ex}$ & Early positivity \\
\hline OS-MMC & + nom & + nom & $-\operatorname{lex}$ & $+\mathrm{acc}$ & $-\operatorname{lex}$ & Early positivity (reduced) \\
\hline OS-MML & + nom & $+\mathrm{acc}$ & $-1 \mathrm{ex}$ & + acc & + lex & - \\
\hline
\end{tabular}

Note. $\quad \mathrm{NP}=$ noun phrase; Intrasent. = intrasentential; intersent. = intersentential; Lex = lexically corresponding to an argument in the context sentence; $\mathrm{SO}=$ subject initial; OS = object initial; NEUT = neutral; $\mathrm{TOP}=$ topic; $\mathrm{FOC}=$ focus; nom $=$ nominative; acc $=$ accusative; $\mathrm{MMC}=$ case mismatch; $\mathrm{MML}=$ lexical mismatch. 


\section{Context Effects for SO Conditions}

For the SO conditions, no intrasentential processing problems are to be expected because SO sentences are generally the unmarked form. Thus, the processing patterns observable here must all be due to the interaction with the context. At the position of NP1, the data reveal a positivity in two conditions, namely in the focus condition (SO-FOC) and in the case mismatch condition (SO-MMC). The positivity in the focus condition (SO-FOC) indicates that this component is somehow associated with the integration of a constituent that is predicted by the question context. It cannot simply reflect the processing of new information (e.g., establishing a new discourse referent), because the neutral condition (SO-NEUT) shows no positivity compared with the topic condition (SO-TOP). Rather, the early positivity must be indicative of processing mechanisms associated with a contextually required element, thus supporting the assumption that some kind of special processing takes place for elements that are explicitly required by the context (cf. Altmann et al., 1998). Because the early positivity is observable for the condition SO-FOC, in which all predictions are fulfilled at the position of NP1 (cf. Table 2), it appears that this component may be viewed as a marker of successful contextual integration.

To shed light on the features involved in this contextual integration, we examined two conditions in which context and target sentence do not match perfectly (i.e., SO-MML/MMC). Here, only $\mathrm{SO}-\mathrm{MMC}$, in which new lexical material bearing a nonpredicted case is encountered at the position of NP1, shows a positivity at the position of the first NP. By contrast, SO-MML, in which the case marking is as predicted, though the lexical material is already given, does not show a positivity. Under an interpretation of the positivity as a marker of successful contextual integration, the introduction of a new referent thus appears more important for this integration process than the fulfillment of a case-related prediction. Recall from above that the two mismatch conditions are not violation conditions and, therefore, the lack of a positivity in SO-MML cannot be attributed to the processing of a violation. ${ }^{6}$

In summary, the positivity for constituents corresponding to the $w h$-pronoun of the context question reflects the fulfillment of a prediction generated by the context. This characterization of the component in question is supported by the fact that the two nonneutral conditions that do not show such a positivity at the position of NP1, namely SO-MML and SO-TOP, show a highly similar effect at the position of NP2. Recall that, in these conditions, it is the second NP rather than the first at which the new lexical material required to saturate the argument slot provided by the context is encountered. Hence, the early positivity appears to reflect processes of focus integration that are highly consistent and generalize over various positions in a sentence.

\section{Context Effects for OS Conditions}

For OS sentences in a neutral context, the present experiment replicated previous findings of a negative ERP component. However, despite the fact that previous behavioral studies have shown that the processing of OS sentences of the type examined here is facilitated in a context in which the initial object is given (Meng et al., 1999), the local negativity was not reduced in this condition (OS-TOP) in the present experiment (i.e., the contextual licensing of an OS order does not suffice to alleviate the local mismatch). Rather, the negativity has a shorter latency when an object has been given than when it is encountered in a neutral context, thus indicating that the processing conflict may be more visible (i.e., easier to recognize) in the former case. With regard to the crucial question of whether contextual information may influence initial processes of syntactic integration, these observations allow two possible conclusions. First, it is possible that contextual information is not accessible early enough to facilitate the integration of an initial object. Alternatively, however, it may be that givenness of an initial object is not a suitable licensing factor with regard to the local mismatch and that licensing takes place at a more global level (i.e., possibly at the level of the clause, rather than of individual sentential constituents), namely by increasing the acceptability of the OS structure (cf. Meng et al., 1999). We attempt to differentiate between these two possible interpretations below.

The crucial question for a possible dissociation between the two accounts outlined in the last paragraph concerns how intersentential and sentence-internal predictions interact in the OS structures. Whereas the SO conditions have shown that the context question generates a prediction that the slot opened by the $w h$-pronoun be saturated, the processing difficulty for (clause-medial) OS sentences has been interpreted as reflecting a local mismatch between the predicted subject position and the element actually encountered in this position. Thus, as indicated in Table 2, the sentenceprocessing system apparently makes two case-related predictions, one contextual and one sentence-internal, both of which apply to the position of the first NP in the present experiment. The diagnostic tools available to us for the examination of how these case-related predictions interact are (a) the positivity observable when a contextual prediction is fulfilled and (b) the negativity reflecting the nonfulfillment of a syntactic prediction.

Table 2 lists early positivities for two OS conditions, namely for the OS-FOC and the OS-MMC conditions (i.e., the same two conditions showing a positivity at NP1 in the SO case), although the positivity for the latter condition is distinctly smaller than that for the former. The difference between the two conditions lies in the degree to which the contextual prediction is fulfilled: Whereas NP1 in OS-FOC corresponds exactly to the element predicted by the context, NP1 in OS-MMC only provides an argument with a new referent but not bearing the expected case. However, because there was no difference between the two analogous SO conditions, the reduced amplitude of the positivity for OS-MMC is apparently crucially tied to the fact that an object needs to be integrated into the current sentence.

\footnotetext{
${ }^{6}$ The fact that there is no positivity at the position of the first NP when this NP is given by the context, although bearing a contextually unpredicted case marking, indicates that the positivity should not be described in terms of the assignment of prosodic focus, because in this condition the first NP must be stressed contrastively: for example, "Wer hat den Jäger beobachtet? Ich glaube, dass der JÄGER den Gärtner beobachtet hat" ("Who watched the hunter? I think that (it was) the HUNTER (who) watched the gardener"). Thus, under a prosodic account, this condition should also show a positivity at the position of the first NP.
} 
This pattern of results indicates that a full match between an initial object and a contextual prediction can (briefly) override sentence-internal (syntactic integration based) concerns, whereas this is not possible when the match between the element and the contextual expectation is not complete. In the latter case, syntactic integration must take place as usual in the sentence currently being processed, thus giving rise to a local syntactic mismatch and negativity in the ERP. However, because new lexical material apparently suffices for integration with the contextually specified slot to take place (as the SO case showed), there is also a positivity reflecting this contextual integration step. On the surface, the interaction of both components results in the apparently smaller amplitude of the positivity in the OS-MMC case.

In this way, the only condition that remains to be accounted for is OS-MML, in which neither a positivity nor a negativity is observable. Essentially, in this condition, there is a contextual expectation for an accusative and new lexical material, but at the position of the first NP only the accusative prediction is fulfilled, because the lexical material encountered there is already given. As the corresponding SO condition showed, there is no contextual integration in this type of constellation. However, why should there be no negativity? A somewhat speculative answer to this question is that this condition may, in fact, constitute the only structure in which the OS order is truly licensed. Here, the prediction for accusative is fulfilled, although with given lexical material, thereby implying a contrastive focus intonation on the initial NP. In this way, an OS structure appears to be licensed when the object is a contrastively focused given element. ${ }^{7}$ However, the present experiment does not allow us to fully draw these conclusions with regard to the OS-MML condition, because no variations with regard to prosodic contour were included.

In summary, the data for the OS sentences show that context may influence even early stages of sentence processing, because contextual requirements can override sentence-internal syntactic requirements under certain circumstances. To be more specific, this claim is based on the finding that an early positivity may supersede a scrambling negativity when the properties of the scrambled object perfectly match the contextual prediction generated by the constituent question. In this way, it appears that contextual information may influence, or even override, sentenceinternal syntactic processing only when this serves to fulfill a contextual prediction.

\section{Functional Characterization of the Early Positivity}

The early positivity observed for focused constituents in the present experiment resembles the $\mathrm{P} 3 \mathrm{~b}$ event-related brain potential with regard to latency, topography, and morphology. At a first glance, characterizing the focus positivity as a P3 component is also quite attractive in terms of the functional interpretation of this component, because the P3 is elicited by the processing of target stimuli and is modulated, among other factors, by informativity and task relevance of a stimulus as well as the degree of its expectedness (cf. Picton, 1993, for an overview). Under such an account, the focus positivity observed here would reflect general cognitive integration costs that arise when a target stimulus is processed. In this particular case, a stimulus is identified as a target by way of the contextually generated expectation for its occurrence.

However, recall that highly similar positivities to those observed at the position of focused arguments were also elicited at the position of the verb in nonneutral contexts. Under the P3 account, this finding is unexpected, because the verb is contextually given in all nonneutral contexts and, hence, cannot qualify as a target stimulus to a greater degree than a given argument. Thus, if all of the early positivities observed in the present study are to be accounted for in a unified way-as, indeed, appears desirable in view of the high degree of similarity between them-it seems that a domain general account does not suffice. Rather, the linguistic properties of the critical constituents must be taken into account.

Note that the proposition expressed by a given sentence can only be fully established with the processing of the verb. Therefore, under the plausible assumption that not only elements predicted by the context but also entire new propositions must be contextually integrated, the distribution of the early positivities in the present experiment may be derived. From this perspective, the focus positivity reflects the comparison of a predicted linguistic constituent-be it at the phrase or at the sentence level-to the contextually generated expectation and the integration of the predicted element into the intersentential context, a mechanism that appears language specific, rather than domain general. ${ }^{8}$

\section{Consequences for Psycholinguistic Models}

Finally, let us consider the consequences of the present data for psycholinguistic theories. As discussed in detail above, our data show a clear interaction between sentence-internal and intersentential processing mechanisms, because there is an interaction between word order and context at the position of the first NP in the target sentence. Thus, context is able to influence initial syntactic integration, contrary to the predictions of strong versions of syntax-first models such as the garden path model (cf. Frazier, 1987).

However, similar to Altmann et al.'s (1998) results, our findings suggest that early contextual influences crucially depend on the specific properties of the context, because only the contextual predictions interacted with sentence-internal processing concerns. The condition in which the initial object of the target sentence was given, although giving rise to higher acceptability ratings (Meng et al., 1999) could not alleviate the local processing difficulties associated with an OS word order in the types of sentences used here, a finding contrary to the predictions of strongly interactive

\footnotetext{
${ }^{7}$ A possible explanation of why a given element may need to be contrastively focused to license an OS structure is that, in contrast to other types of focus, contrastive focus is not relational (i.e., the fact that an element is contrastively focused may be determined by considering this element alone [though in relation to the context], rather than having to take other arguments, for example, into account as well; Féry, 1993).

${ }^{8}$ These arguments do not fully exclude the possibility that the early positivity is an instantiation of the $\mathrm{P} 3$, because one might consider the entire clause a target constituent from this perspective. However, such an interpretation would crucially require the P3 to be interpreted as a component sensitive to linguistic categories.
} 
processing models (e.g., MacDonald, Pearlmutter, \& Seidenberg, 1994).

Thus, one may conclude from these results that the parser certainly has access to contextual information-at least of the predictive type-at even the earliest stages of syntactic integration but that this information only influences early sentence-internal processes when a particular contextual requirement (prediction) explicitly calls for an integration between the context and certain constituents or properties of the target sentence.

\section{References}

Altmann, G. T. M. (1999). Thematic role assignment in context. Journal of Memory and Language, 41, 124-145.

Altmann, G. T. M., Garnham, A., \& Dennis, Y. I. L. (1992). Avoiding the garden path: Eye movements in context. Journal of Memory and Language, $31,685-712$

Altmann, G. T. M., Garnham, A., \& Henstra, J. A. (1994). Effects of syntax in human sentence parsing: Evidence against a structure-based proposal mechanism. Journal of Experimental Psychology: Learning, Memory, and Cognition, 20, 209-216.

Altmann, G. T. M., \& Steedman, M. (1988). Interaction with context during human sentence processing. Cognition, 30, 191-238.

Altmann, G. T. M., van Nice, K. Y., Garnham, A., \& Henstra, J.-A. (1998). Late closure in context. Journal of Memory and Language, 38, 459484

Bornkessel, I., Schlesewsky, M., \& Friederici, A. D. (2002). Grammar overrides frequency: Evidence from the online processing of flexible word order. Cognition, 85, B21-B30.

Britt, M. A., Perfetti, C. A., Garrod, S., \& Rayner, K. (1992). Parsing in discourse: Context effects and their limits. Journal of Memory and Language, 31, 293-314.

Crain, S., \& Steedman, M. (1985). On not being led up the garden path: The use of context in the psychological syntax processor. In D. R. Dowty, L. Kartunnen, \& A. M. Zwicky (Eds.), Natural language parsing (pp. 320-358). Cambridge, England: Cambridge University Press.

Ferreira, F., \& Clifton, C., Jr. (1986). The independence of syntactic processing. Journal of Memory and Language, 25, 348-368.

Féry, C. (1993). German intonation patterns. Tübingen, Germany: Niemeyer.

Frazier, L. (1987). Sentence processing: A tutorial review. In M. Coltheart (Ed.), Attention and performance: Vol. 12. The psychology of reading (pp. 559-586). Hove, England: Erlbaum.

Friederici, A. D. (1999). The neurobiology of language processing. In A.D. Friederici (Ed.), Language comprehension: A biological perspective (pp. 265-304). Berlin/Heidelberg/New York: Springer.

Friederici, A. D. (2002). Towards a neural basis of auditory sentence processing. Trends in Cognitive Sciences, 6, 78-84.

Friederici, A. D., \& Frisch, S. (2000). Verb-argument structure processing: The role of verb-specific and argument-specific information. Journal of Memory and Language, 43, 476-507.

Friederici, A. D., Schlesewsky, M., \& Fiebach, C. J. (2003). Wh-movement vs. scrambling: The brain makes a difference. In S. Karimi \& $\mathrm{T}$. Langendoen (Eds.), Word order and scrambling (pp.325-344). Oxford, England: Blackwell.

Hagoort, P., \& Brown, C., \& Groothusen, J. (1993). The syntactic positive shift as an ERP-measure of syntactic processing. Language and Cognitive Processes, 8, 439-483.

Huynh, H., \& Feldt, L. S. (1970). Conditions under which the mean square ratios in repeated measurement designs have exact $F$-distributions. Journal of the American Statistical Association, 65, 1582-1589.

Keller, F., \& Alexopoulou, T. (2001). Phonology competes with syntax: Experimental evidence for the interaction of word order and accent placement in the realization of information structure. Cognition, 79, 301-372.

Keppel, G. (1991). Design and analysis (3rd ed.). Englewood Cliffs, NJ: Prentice Hall.

Kiss, K. E. (1995). Discourse configurational languages. Oxford, England: Oxford University Press.

Kutas, M., \& Hillyard, S. A. (1980). Reading senseless sentences: Brain potentials reflect semantic incongruity. Science, 207, 203-205.

Kutas, M., \& Hillyard, S. A. (1983). Event-related potentials to grammatical errors and semantic anomalies. Memory \& Cognition, 11, 539-550.

Lenerz, J. (1977). Zur Abfolge nominaler Satzglieder im Deutschen [On the order of nominal sentence constituents]. Tübingen, Germany: Narr.

Liversedge, S. P., Pickering, M. J., Branigan, H. P., \& van Gompel, R. P. G. (1998). Processing arguments and adjuncts in isolation and context: The case of by-phrase ambiguities in passives. Journal of Experimental Psychology: Learning, Memory, and Cognition, 24, 461475.

MacDonald, M. C., Pearlmutter, N. J., \& Seidenberg, M. S. (1994). The lexical nature of syntactic ambiguity resolution. Psychological Review, 101, 676-703.

Meng, M., Bader, M., \& Bayer, J. (1999). Die Verarbeitung von SubjektObjekt Ambiguitäten im Kontext [The processing of subject-object ambiguities in context]. In I. Wachsmuth \& B. Jung (Eds.), Proceedings der 4. Fachtagung der Gesellschaft für Kognitionswissenschaft (pp. 244-249). St. Augustin, Germany: Infix Verlag.

Mitchell, D. C., Corley, M. M. B., \& Garnham, A. (1992). Effects of context in human sentence parsing: Evidence against a discourse-based proposal mechanism. Journal of Experimental Psychology: Learning, Memory, and Cognition, 18, 69-88.

Murray, W. S., \& Liversedge, S. P. (1994). Referential context effects on syntactic processing. In C. Clifton, L. Frazier, \& K. Rayner (Eds.), Perspectives on sentence processing (pp. 359-388). Hillsdale, NJ: Erlbaum.

Neville, H. J., Nicol, J., Barss, A., Forster, K., \& Garrett, M. F. (1991), Syntactically based sentence processing classes: Evidence from eventrelated potentials. Journal of Cognitive Neuroscience, 6, 233-255.

Osterhout, L., \& Holcomb, P. J. (1992). Event-related brain potentials elicited by syntactic anomaly. Journal of Memory and Language, 31, 785-804.

Osterhout, L., \& Holcomb, P. J. (1993). Event-related brain potentials and syntactic anomaly: Evidence of anomaly detection during the perception of continuous speech. Language and Cognitive Processes, 8, 413-437.

Picton, T. W. (1993). The P300 wave of the human event-related brain potential. Journal of Clinical Neurophysiology, 9, 456-479.

Rayner, K., Garrod, S., \& Perfetti, C. A. (1992). Discourse influences during parsing are delayed. Cognition, 45, 109-139.

Rösler, F., Pechmann, T., Streb, J., Röder, B., \& Hennighausen, E. (1998). Parsing of sentences in a language with varying word order: Word-byword variations of processing demands are revealed by event-related brain potentials. Journal of Memory and Language, 38, 150-176.

Schlesewsky, M., Bornkessel, I., \& Frisch, S. (2003). The neurophysiological basis of word order variations in German. Brain and Language, $86,116-128$

Trueswell, J. C., \& Tanenhaus, M. K. (1991). Tense, temporal context and syntactic ambiguity resolution. Language and Cognitive Processes, 6 , 303-338.

van Berkum, J. J. A., Brown, C. M., \& Hagoort, P. (1999). Early referential context effects in sentence processing: Evidence from event-related brain potentials. Journal of Memory and Language, 41, 147-182.

Received July 3, 2002

Revision received February 5, 2003

Accepted February 9, 2003 